\title{
Posaconazole: A new agent for the prevention and management of severe, refractory or invasive fungal infections
}

\author{
Andrea V Page MD FRCPC ${ }^{1,2}$, W Conrad Liles MD PhD FRCPC ${ }^{1,2}$
}

\begin{abstract}
AV Page, WC Liles. Posaconazole: A new agent for the prevention and management of severe, refractory or invasive fungal infections. Can J Infect Dis Med Microbiol $2008 ; 19(4): 297-305$.

Posaconazole is the newest antifungal agent to be approved for use in Canada. With excellent in vitro activity against a broad spectrum of yeasts and filamentous fungi, as well as having a well-tolerated oral formulation, posaconazole offers many potential advantages. Of particular interest are its seemingly lower potential for cross-resistance with other azoles and its activity (unique among oral antifungal agents) against the zygomycetes. As the incidence of both common and uncommon fungal infections increases commensurate with the growing population of immunocompromised individuals, posaconazole may ultimately become an important therapeutic option. The present article reviews the in vitro and in vivo data describing its activity, and focuses on both the proven and the potential clinical applications of this new triazole agent.
\end{abstract}

Key Words: Aspergillosis; Candidiasis; Mucormycosis; Posaconazole; Zygomycosis

Severe and invasive fungal infections (IFIs) are increasing in Sincidence. Intensive chemotherapeutic regimens, hematopoietic stem cell and solid organ transplantations, corticosteroid and biological therapies, and HIV/AIDS have all contributed to the growing number of at-risk immunosuppressed individuals. In this patient population, serious fungal infections range from recurrent and potentially debilitating infections (refractory oropharyngeal candidiasis [OPC]) to acute and potentially fatal infections (invasive aspergillosis or zygomycosis following transplantation). IFIs portend a particularly poor prognosis in certain high-risk subpopulations; in two large, retrospective studies $(1,2)$ of allogeneic hematopoietic stem cell transplant (HSCT) recipients, attributable mortality rates varied between $20 \%$ for those patients with candidemia and $77.2 \%$ for those with invasive aspergillosis.

These studies of IFI-associated mortality were conducted either in full or in part during an era in which antifungal therapy was limited to the polyenes, such as amphotericin B, or the early generation triazoles, such as fluconazole or itraconazole. Amphotericin B, whether in the deoxycholate or lipid-based formulation, is an intravenous medication with the most diverse spectrum of activity of any antifungal agent. However, its use is associated with a high rate of infusion reactions and nephrotoxicity; progressive fungal disease has occurred in patients who were unable to complete treatment (3). In addition, fungi
Le posaconazole : Un nouvel agent pour prévenir et prendre en charge les infections à champignons graves, réfractaires ou envahissantes

Le posaconazole est le dernier antifongique à être approuvé au Canada. Étant donné son excellente activité in vitro contre un large spectre de levures et de champignons filamenteux et sa formulation orale bien tolérée, le posaconazole offre de nombreux bienfaits potentiels. Notamment, il a un potentiel en apparence plus faible à l'interrésistance à d'autres azoles et une activité (unique parmi les antifongiques oraux) contre les zygomycètes. Puisque l'incidence des infections à champignons courantes et peu courantes augmente proportionnellement avec la population de personnes immunocompromises, le posaconazole pourrait bien devenir une option thérapeutique importante. Le présent article analyse les données in vitro et in vivo décrivant son activité et porte à la fois sur les applications cliniques démontrées et potentielles de ce nouveau triazole.

resistant to amphotericin B, such as Aspergillus terreus, have emerged as important human pathogens (4).

Although generally better tolerated than amphotericin B, particular characteristics of the early generation triazole agents also limit their use: fluconazole has no activity against certain Candida species and most filamentous fungi, while itraconazole has multiple drug-drug interactions and variable bioavailability, particularly in capsule form.

However, in recent years, a spate of new antifungal options, including the expanded-spectrum triazoles (such as voriconazole) and the echinocandins have emerged. Both are welltolerated and active against most Candida and Aspergillus species. However, both also lack activity against notable causes of post-transplantation IFIs, including the zygomycetes and Fusarium species. Voriconazole prophylaxis in HSCT recipients has been contemporaneously associated with an increase in infections due to these organisms, which hints at the potential clinical relevance of these omissions from the spectrum of activity of any antifungal agent $(5,6)$. In addition, case reports (7-9) have documented the emergence of echinocandin resistance during treatment of candidemia.

An appraisal of the properties of these existing agents suggests that a well-tolerated oral medication with a broad spectrum of activity against both yeasts and filamentous fungi, as well as a high barrier to the development of resistance, may

${ }^{1}$ Division of Infectious Diseases, University Health Network, ${ }^{2}$ Department of Medicine, University of Toronto, Toronto, Ontario

Correspondence: Dr Andrea V Page, University Health Network, Toronto General Hospital, 13 Eaton North, Room 208, 200 Elizabeth Street,

Toronto, Ontario M5G 2C4. Telephone 416-340-4800 ext 2197, fax 416-340-3357, e-mail andrea.page@uhn.on.ca

Received for publication January 8, 2008. Accepted June 2, 2008 
TABLE 1

Comparative in vitro spectra of activity of antifungal agents currently approved for use in Canada

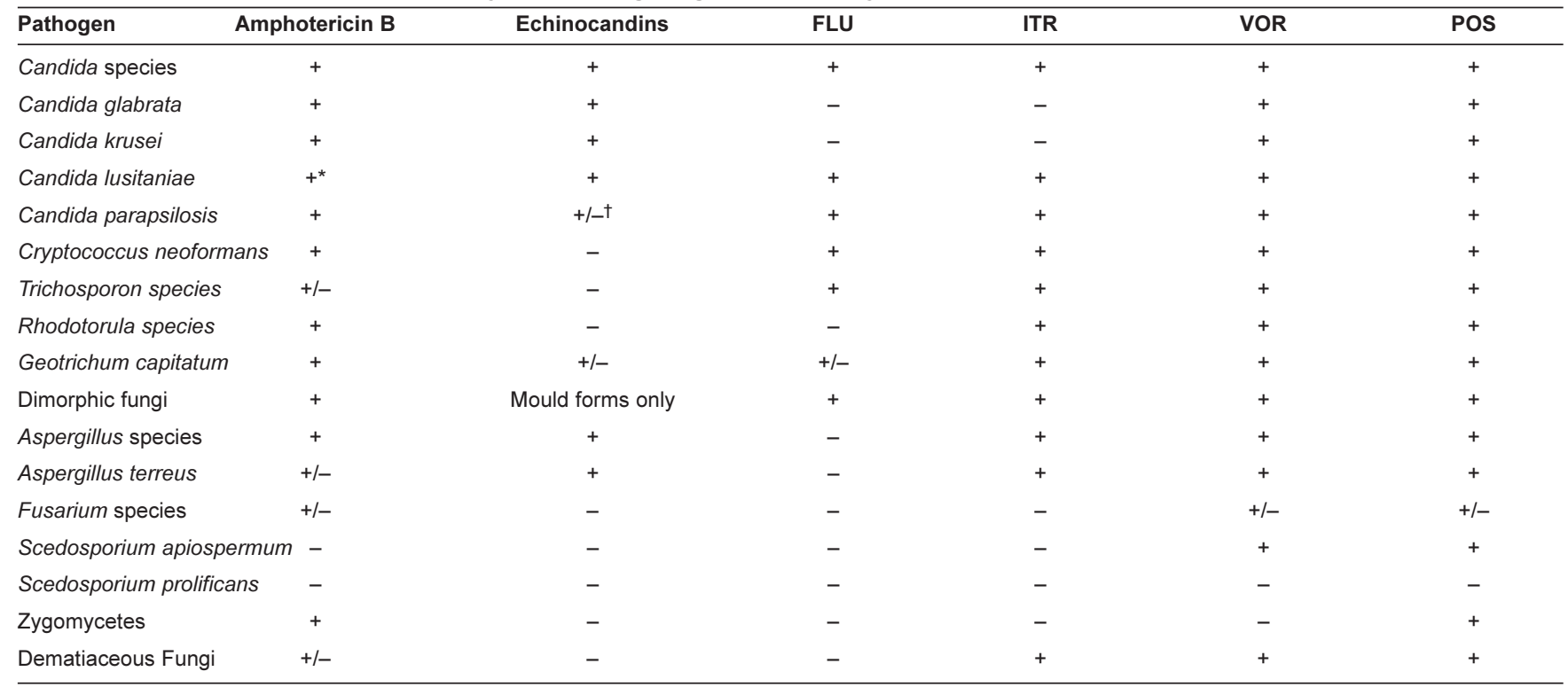

${ }^{*}$ Resistance may develop during treatment; ${ }^{\dagger}$ The minimum concentration of posaconazole (POS) required to inhibit the growth of $90 \%$ of isolates was greater than $16 \mathrm{mg} / \mathrm{L}$ in some studies. FLU Fluconazole; ITR Itraconazole; VOR Voriconazole. Data from references 63 to 67

provide a useful therapeutic option. Approved for use in Canada in 2007, posaconazole possesses an in vitro spectrum of activity comparable with amphotericin B (Table 1), an oral formulation and a low risk of adverse events. It has been granted Health Canada approval for the treatment of OPC and refractory invasive aspergillosis, as well as for the prophylaxis of invasive aspergillosis and candidiasis in certain high-risk patient populations (10). In Europe, however, its indications for use are broader, and include refractory fusariosis, chromoblastomycosis, mycetoma and coccidioidomycosis (11). The present article reviews the evidence in support of the current Canadian indications for use of posaconazole and explores its potential clinical applications for the treatment of a wide variety of other opportunistic mycoses.

\section{MECHANISM OF ACTION}

Similiar to all triazoles, posaconazole inhibits the fungal lanosterol 14 alpha-demethylase enzyme (CYP51), a member of the cytochrome P450 family (12). In doing so, posaconazole blocks the synthesis of ergosterol, the primary sterol in the fungal cell membrane, and an important determinant of cell membrane integrity, membrane-associated protein function and progression through the cell cycle.

\section{PHARMACOKINETICS}

Posaconazole is administered as an oral suspension at a dose of $800 \mathrm{mg}$ per day; no parenteral formulation is currently available. Serum drug concentrations increase in a dose-proportional manner up to the recommended daily dose, with no further increases thereafter (13). Because high-fat meals, nutritional supplements and frequent administration all promote the absorption of posaconazole, the $800 \mathrm{mg}$ dose is divided twice daily if the patient is taking enteral nutrition, and four times daily if not $(14,15)$. In fasting individuals, the average steady-state plasma concentration is $0.5 \mathrm{mg} / \mathrm{L}$ based on four times daily dosing; it is slightly higher at $0.72 \mathrm{mg} / \mathrm{L}$ when administered twice daily with a high-fat meal or nutritional supplement $(10,15)$. In contrast, voriconazole administered at the recommended treatment dose of $200 \mathrm{mg}$ twice daily achieves average plasma concentrations of $2.5 \mathrm{mg} / \mathrm{L}$ (16). When posaconazole was studied for antifungal prophylaxis in patients with chemotherapy-induced neutropenia or severe graft-versus-host disease, the recommended dose of $200 \mathrm{mg}$ three times daily achieved a mean steady-state plasma concentration of $0.58 \mathrm{mg} / \mathrm{L}$, albeit with a wide range of concentrations up to a maximum of $1.47 \mathrm{mg} / \mathrm{L}(17,18)$. Notably, an association between lower average plasma concentrations of posaconazole and treatment failures has been reported (19).

As a liquid suspension, posaconazole has significantly greater bioavailability than as a tablet and reaches its peak plasma concentration in $10 \mathrm{~h}$ (20). The volume of distribution of posaconazole, at least 10-fold greater than the total body water content, suggests a wide distribution throughout peripheral tissues. In particular, posaconazole has been shown to be at least as effective as amphotericin B in murine and human treatment trials for IFIs of the central nervous system, providing indirect evidence for drug delivery across the blood-brain barrier (21-23). Case reports (24) have also shown drug penetration into the vitreous fluid during the treatment of ocular fungal infections.

Posaconazole has a half-life of $35 \mathrm{~h}$, and is eliminated unchanged through the gastrointestinal tract. Because the kidneys play a minor role in the excretion of posaconazole, no dose adjustment is necessary, even in cases of severe renal impairment (creatinine clearance lower than $20 \mathrm{~mL} / \mathrm{min}$ ). Consistent with the high degree of protein binding (98\%), the drug is not removed by hemodialysis (25). Dose adjustments due to hepatic dysfunction have not been well studied, and many clinical trials have excluded patients with baseline elevations in serum hepatic aminotransferases.

Posaconazole's pharmacokinetic characteristics are not affected by sex, ethnicity or age (26). As with most other triazole 
agents, posaconazole is categorized as pregnancy risk class C, because skeletal malformations were detected in the fetuses of pregnant rats administered 1.4 times the usual adult dose of the drug (10). In a small study (27) of children younger than 18 years of age, all of whom received $800 \mathrm{mg}$ per day of posaconazole as salvage therapy for IFIs, pharmacokinetic parameters, adverse events and treatment outcomes among the children approximated those reported in adults.

Posaconazole inhibits the hepatic CYP3A4 enzyme, a member of the cytochrome P450 enzyme system; therefore, it may raise the serum concentrations of drugs with which it is coadministered, including tacrolimus and cyclosporine (28). Seizures have occasionally occurred when these drugs have been used in combination, underlining the importance of therapeutic drug monitoring (29). Likewise, the concentrations of phenytoin, rifabutin and midazolam may be increased by coadministration with posaconazole (10). Although not yet studied in vitro or in vivo, the concentrations of other CYP3A4 substrates, such as the ergot and vinca alkaloids, sirolimus, the HMG-CoA reductase inhibitors and some calcium channel blockers, may be affected by concomitant administration of posaconazole, and may therefore require dose adjustment and careful monitoring. Coadministration of posaconazole and the ergot alkaloids, or posaconazole and medications which prolong the QT interval, is not recommended (10). Posaconazole has not been found to inhibit the other P450 isoforms studied (30). Although posaconazole is both an inhibitor of and a substrate for P-glycoprotein in vitro, polymorphisms of P-glycoprotein have no effect on posaconazole disposition in vivo $(10,26)$.

\section{ANTIFUNGAL ACTIVITY IN EXPERIMENTAL MODELS, IN VITRO AND IN VIVO}

Posaconazole possesses antifungal activity that is greater than, or within one dilution of, the other triazole agents and amphotericin B against yeasts, dimorphic fungi and most genera of filamentous fungi when examined in vitro (31). The minimum concentration of posaconazole required to inhibit the growth of $90 \%$ of isolates $\left(\mathrm{MIC}_{90}\right)$ was $1 \mathrm{mg} / \mathrm{L}$ when tested against 18,351 yeasts and 4499 hyaline moulds. In comparison, the mean $\mathrm{MIC}_{90}$ values for voriconazole were $0.5 \mathrm{mg} / \mathrm{L}$ and $2.0 \mathrm{mg} / \mathrm{L}$, respectively, when tested against the same isolates.

Posaconazole is potentially fungicidal in vitro against some species of Candida, Cryptococcus neoformans, Trichosporon species, Coccidioides immitis, Histoplasma capsulatum, Blastomyces dermatitidis, Aspergillus species and selected dermatophytes (21,32-37). It is particularly active against yeasts. Posaconazole inhibited $97 \%$ of all Candida species and $98 \%$ of all $\mathrm{C}$ neoformans isolates at a concentration of $1 \mathrm{mg} / \mathrm{L}$ in a large study (38) of over 4000 clinical specimens. The major exception occurred among isolates of Candida glabrata, of which $80 \%$ were inhibited at a concentration of $1 \mathrm{mg} / \mathrm{L}$. By comparison, voriconazole inhibited $92 \%$ of C glabrata isolates at the same concentration. For all other isolates tested, posaconazole was equivalent to voriconazole, inhibiting the same percentage of isolates at concentrations of $1 \mathrm{mg} / \mathrm{L}$. In comparison with fluconazole, posaconazole inhibited a greater percentage of isolates of C glabrata, Candida parapsilosis, Candida tropicalis and Candida krusei at a concentration of $1 \mathrm{mg} / \mathrm{L}$ (38). However, because the mean steady-state plasma concentration of posaconazole achievable in humans was below $1 \mathrm{mg} / \mathrm{L}$, albeit with wide individual variation, these in vitro results suggest that voriconazole may be a more reliable choice for the treatment of confirmed invasive candidiasis; the $\mathrm{MIC}_{90}$ value of voriconazole for most Candida species lies well within the average steady-state plasma concentration achieved with the recommended dosing regimen.

In contrast, posaconazole is more active in vitro against a wide variety of filamentous fungi than is voriconazole. At a concentration of $1 \mathrm{mg} / \mathrm{L}$, posaconazole inhibited $95 \%$ of all 448 isolates of filamentous fungi tested, including the agents of chromoblastomycosis, mycetoma and phaeohyphomycosis, while voriconazole inhibited $90 \%$ of these isolates at the same concentration (39). Although approximately equivalent to voriconazole against most Aspergillus species, particularly the relatively amphotericin-resistant A terreus, posaconazole displayed greater activity against Aspergillus niger (40). In addition, posaconazole was more active than voriconazole against the zygomycetes, more active than amphotericin B against A terreus, and more active than both against Penicillium species and Paecilomyces species $(31,39)$.

Despite these promising in vitro results, there are some notable gaps in the antifungal activity of posaconazole. Although it is the only triazole agent with consistent activity against the zygomycetes, posaconazole displays higher $\mathrm{MIC}_{90}$ values than amphotericin B for Rhizopus, Mucor and Cokeromyces species. In addition, posaconazole has an $\mathrm{MIC}_{90}$ of $16 \mathrm{mg} / \mathrm{L}$ against Cladosporium species, compared with $4 \mathrm{mg} / \mathrm{L}$ for amphotericin B (31). The in vitro activity of posaconazole against all Fusarium species is comparable with that of amphotericin B (both have mean $\mathrm{MIC}_{90}$ values of $32 \mathrm{mg} / \mathrm{L}$ ), but the activity of both is highly species dependent. Similarly, no existing antifungal agent had an $\mathrm{MIC}_{90}$ value less than that of posaconazole, at $32 \mathrm{mg} / \mathrm{L}$, for Scedosporium prolificans.

In vivo experimental animal models of IFIs have uniformly corroborated these in vitro findings. Posaconazole has been shown to improve survival, reduce fungal burden, or both, in disseminated candidiasis; cerebral and disseminated cryptococcosis; cerebral, pulmonary and disseminated aspergillosis; pulmonary blastomycosis; disseminated coccidioidomycosis; disseminated histoplasmosis; cerebral and disseminated phaeohyphomycosis; and disseminated pseudallescheriasis (40). Gaps in the in vivo antifungal activity of posaconazole are the same as those identified by in vitro susceptibility testing; posaconazole demonstrates dose-dependent activity in murine models of disseminated fusariosis and zygomycosis, but no activity in murine models of disseminated infection due to S prolificans (40).

\section{CLINICAL EFFICACY - TREATMENT TRIALS}

\section{Mucosal and invasive candidiasis}

Posaconazole has been most extensively studied for the treatment of mucosal candidiasis in the setting of advanced HIV disease. As primary therapy, posaconazole was compared with fluconazole (200 $\mathrm{mg}$ on the first day, followed by $100 \mathrm{mg}$ for the next 13 days for both drugs) in a randomized, evaluatorblinded study (41) of 350 patients with OPC, most of whom were infected with fluconazole-susceptible strains of Candida albicans. By day 14, an equal number of patients in each group had achieved a satisfactory clinical response (cure or symptomatic improvement in 92\%) or mycological cure (68\%). Although more patients in the posaconazole group had a sustained mycological cure four weeks after the end of treatment, the rate of clinical relapse was not significantly different 
between the two groups $(31.5 \%$ in the posaconazole arm and $38.2 \%$ in the fluconazole arm).

As salvage therapy, posaconazole was evaluated in an observational study (42) of 199 patients with advanced HIV disease and OPC or esophageal candidiasis that had failed to respond to a standard duration of fluconazole or itraconazole therapy (10 days for OPC and three weeks for esophageal candidiasis). Relapsing mucosal candidiasis was a common occurrence in patients with advanced HIV disease before the advent of highly active antiretroviral therapy. With the widespread use of antiretroviral therapy, treatment-refractory mucosal candidiasis is now observed much less frequently, occurring in approximately $4 \%$ of patients with a history of OPC and with CD4 counts less than 150 cells/ $\mu \mathrm{L}$ (43). Fluconazole-resistant Candida species colonize the oropharynx in $8 \%$ of fluconazole-naive patients with HIV and in $45 \%$ of fluconazole-experienced patients with $\operatorname{HIV}(43,44)$. In the present study of posaconazole salvage therapy, the infecting species of Candida was resistant to fluconazole and/or itraconazole in the majority of patients. Eligible patients received posaconazole according to a schedule of either $800 \mathrm{mg}$ per day for three days followed by $400 \mathrm{mg}$ per day for 25 days or $800 \mathrm{mg}$ per day for 28 days. The primary end point of cure or improvement after 28 days of therapy was met in $75 \%$ of participants. Furthermore, the same degree of success was observed in the subset of patients infected with fluconazoleand/or itraconazole-resistant Candida species. These results are comparable with those achieved with caspofungin, the other agent approved for use in the treatment of refractory mucosal candidiasis (45). Notably, $74 \%$ of patients overall (80\% receiving the low-dose posaconazole regimen and $68 \%$ receiving the standard-dose regimen) had recurrent signs and/or symptoms four weeks after the end of treatment.

To combat the high relapse rate following discontinuation of treatment, Vazquez et al (46) investigated the suitability of posaconazole (800 mg/day) for use as chronic suppressive therapy for azole-refractory mucosal candidiasis in patients with HIV. In this open-label, noncomparative study, 77 of 90 patients $(85.6 \%)$ in the modified intention-to-treat population were considered to have had a complete or partial clinical response after receiving posaconazole for up to three months. Fifteen patients continued therapy for a full 12 months. Of the 28 patients who discontinued treatment at or before the threemonth evaluation, $13(46.4 \%)$ experienced a relapse within one month after discontinuing therapy. No posaconazole susceptibility data were reported on isolates from those patients who failed therapy or relapsed after discontinuation of therapy. Of those who continued treatment beyond three months, 25\% experienced a treatment-related adverse event. Eight patients discontinued therapy as a result of adverse events. While the present study suggests that posaconazole is effective as chronic suppressive therapy for refractory mucosal candidiasis in the select subpopulation of patients who can tolerate long-term treatment, almost one-third of the modified intention-to-treat population discontinued therapy within three months, and almost $50 \%$ of these patients had a clinical relapse within one month of treatment discontinuation. Longer posttreatment follow-up is required to determine the true benefit, if any, of extended courses of therapy.

Nonetheless, based on the data from the primary and salvage therapy studies, posaconazole has been approved in Canada for the treatment of OPC in patients 13 years of age and older. Posaconazole is unlikely to find immediate use as first-line therapy, however, given that fluconazole is well tolerated, inexpensive and effective for most patients. On the other hand, posaconazole's oral formulation does offer significant benefit over intravenous caspofungin for the treatment of azole-refractory mucosal candidiasis.

In contrast, posaconazole is neither approved nor well studied for the treatment of invasive candidiasis. In the largest trial (29) of posaconazole therapy involving 362 patients, of whom the majority had an underlying malignancy or had undergone hematopoietic stem cell or solid organ transplantation, 39 patients had refractory invasive candidiasis. However, details of the indications for treatment and treatment outcomes are lacking. There are two case reports $(47,48)$ of posaconazole salvage therapy in patients with acute myelogenous leukemia - one patient had disseminated $\mathrm{C}$ albicans infection and the other had $\mathrm{C}$ krusei vertebral osteomyelitis. Both infections were successfully eradicated after prolonged posaconazole therapy (10 and 12 months, respectively). Although these reports suggest that posaconazole may also be effective as salvage therapy for invasive candidiasis, more data are needed before posaconazole gains widespread use for this indication.

\section{Invasive aspergillosis}

Posaconazole has also been evaluated as salvage therapy in a study of 107 patients with invasive aspergillosis, most of whom had an underlying hematologic malignancy or had undergone HSCT (19). Eighty-eight per cent of patients were considered to be refractory to conventional antifungal therapy; the majority having received either amphotericin B or itraconazole for a minimum of seven days without clinical improvement. The remainder were unable to tolerate standard therapy. Pulmonary aspergillosis was the primary diagnosis in $74 \%$ of patients. When compared with 86 matched control subjects with invasive aspergillosis who had been treated with either amphotericin B or itraconazole as salvage therapy, significantly more patients receiving posaconazole had a complete or partial response than those receiving standard salvage therapy (42\% versus $26 \%$, adjusted OR 4.06). This response was consistent regardless of the site of infection or the infecting Aspergillus species, but was significantly greater in the subgroup of patients who achieved average plasma drug concentrations higher than $1 \mathrm{mg} / \mathrm{L}$ as a result of coadministration of posaconazole with food or a nutritional supplement. Survival rates at both 30 days and the end of therapy also differed between the two treatment arms, being $74 \%$ and $38 \%$ in the posaconazole-treated patients, and $49 \%$ and $22 \%$ in the patients receiving standard salvage therapy, respectively.

Posaconazole was also compared directly with high-dose lipid formulation amphotericin B (greater than or equal to $7.5 \mathrm{mg} / \mathrm{kg}$ per day), as well as with a combination of high-dose lipid formulation amphotericin $\mathrm{B}$ and caspofungin in a retrospective study (49) of salvage therapy for proven or probable invasive aspergillosis in HSCT recipients and patients with hematologic malignancies. Primary antifungal therapy consisted of lipid formulation amphotericin B monotherapy in more than $50 \%$ of the patients, while only four of 143 patients overall, and none in the posaconazole group, received primary therapy with voriconazole. Significantly more patients in the posaconazole arm demonstrated a complete or partial response to treatment than 
patients in either of the amphotericin arms when assessed at the end of therapy or after 12 weeks, whichever came first (40\% versus $8 \%$ and $11 \%$ for amphotericin B alone and in combination with caspofungin, respectively; $\mathrm{P}<0.01)$. Importantly, patients in both amphotericin arms had significantly fewer days of therapy (mean duration 16 to 18 days in the amphotericin arms versus 70 days in the posaconazole arm; $\mathrm{P}<0.001)$. In addition, $15 \%$ of patients in the amphotericin monotherapy arm, and $42.1 \%$ of patients in the amphotericin and caspofungin combination arm, were infected with amphotericin-resistant A terreus. Any or all of these factors may have contributed to the observed superiority of posaconazole over the comparator regimens.

Nonetheless, whether secondary to greater antifungal activity or greater tolerability, these data support a role for posaconazole as second-line therapy in the treatment of immunocompromised patients with invasive aspergillosis. The results are comparable with those obtained when caspofungin monotherapy is used for this indication (50). Notably, however, few patients in either posaconazole trial had received voriconazole as primary therapy. Therefore, the role of posaconazole as a second triazole for salvage therapy in this group of patients is still unclear. In addition, there have been no human treatment trials investigating posaconazole as primary therapy for invasive aspergillosis, nor comparing it directly to voriconazole. Posaconazole, therefore, has a very specific indication for the treatment of invasive aspergillosis that is refractory to amphotericin B or itraconazole (progression or failure to improve after seven days), or that occurs in patients intolerant of these agents. It is not approved for use as first-line therapy for invasive aspergillosis.

\section{Other IFIs}

Multiple small observational studies have been conducted to investigate the use of posaconazole as salvage therapy in less common mycoses (Table 2). As second-line therapy, posaconazole has been used successfully to treat mucormycosis, fusariosis, phaeohyphomycosis, eumycetoma and chromoblastomycosis, histoplasmosis and coccidioidomycosis. Although the rates of clinical success, usually defined as either a partial or complete clinical response, were comparable in all studies with those achieved with accepted standard therapy, it should be noted that no study directly compared posaconazole with another antifungal agent. However, given the relative rarity of these infections, randomized trials are likely to be difficult to perform and may not be forthcoming.

There is a single published case report (51) of successful posaconazole salvage therapy for paecilomycosis in a 60 -year-old man with multiple myeloma in whom chronic suppressive therapy with posaconazole facilitated successful allogeneic HSCT. Posaconazole use for the treatment of other agents of hyalohyphomycosis has not been reported.

Posaconazole is of particular interest in the treatment of mucormycosis, for which amphotericin B remains the only other therapeutic option. Although posaconazole has never been compared directly with amphotericin B in vivo, it has been evaluated as salvage therapy for mucormycosis in 91 patients, the majority of whom had an underlying hematologic malignancy or had undergone hematopoietic stem cell transplantation (52). Almost one-half of the studied patients had received prior antifungal prophylaxis with an azole, and in particular, $20 \%$ had received voriconazole.
Eighty per cent of patients received posaconazole for at least 30 days (range six to 1005 days), and 64 of 90 patients also underwent surgical debridement. Twelve weeks after initiation of posaconazole, $60 \%$ of patients had either a complete or partial response to therapy. Notably, however, only $14 \%$ of patients were considered to have had a complete response, and at least some patients were still receiving posaconazole at the time of evaluation. Nonetheless, the low risk of adverse events makes posaconazole an attractive option in the management of mucormycosis, particularly when compared with amphotericin B.

As primary therapy, posaconazole has been investigated for use in the treatment of pulmonary and disseminated (nonmeningeal) coccidioidomycosis (53). While considered successful in $85 \%$ of patients at the end of treatment, chronic posaconazole therapy was often required to prevent relapse. As is frequently the case with less common IFIs, the necessary duration of treatment with posaconazole is unclear and has varied widely among published reports.

As a result of these studies, posaconazole has received approval from the European Medicines Agency for the treatment of refractory fusariosis, chromoblastomycosis, mycetoma and coccidioidomycosis (11). It is not approved in Canada or the United States for these indications.

\section{CLINICAL EFFICACY - PROPHYLAXIS TRIALS}

Given the increasing incidence of IFIs in immunocompromised patients, the role of posaconazole as preventive therapy has also been investigated. In a randomized trial (17) of 602 patients receiving chemotherapy for either acute myelogenous leukemia or myelodysplastic syndrome, prophylaxis with either posaconazole $(200 \mathrm{mg}$ three times daily in 304 patients), fluconazole (400 mg once daily in 240 patients) or itraconazole ( $200 \mathrm{mg}$ twice daily in 58 patients) was given with each cycle of chemotherapy, until either recovery from neutropenia and complete remission, or until 12 weeks following randomization. At the end of treatment, IFIs, as adjudicated by an independent review committee, had occurred in $2 \%$ of patients receiving posaconazole and $8 \%$ of patients receiving either fluconazole or itraconazole $(\mathrm{P}<0.001)$. Twenty patients receiving either fluconazole or itraconazole developed invasive aspergillosis, compared with two patients receiving posaconazole. The number needed to treat with posaconazole as compared with either fluconazole or itraconazole to prevent one IFI was 16 . Patients in the fluconazole or itraconazole arms also received longer courses of systemic antifungal therapy for suspected, but not proven, IFIs. Furthermore, a 33\% relative reduction in mortality 100 days after randomization was noted among patients receiving posaconazole compared with those receiving alternative prophylactic regimens. Note, however, that serious adverse events possibly related to treatment were experienced by $6 \%$ of patients in the posaconazole arm, and by only $2 \%$ in the fluconazole or itraconazole arms $(\mathrm{P}=0.01)$.

In a randomized, double-blind trial (18) of 600 patients receiving intensive immunosuppressive therapy for severe graft-versus-host disease following HSCT, four-month prophylactic regimens of posaconazole and fluconazole were found to be equivalent for the prevention of IFIs and death. However, posaconazole was superior for the prevention of invasive aspergillosis $(\mathrm{OR}=0.31)$ and for a reduction in deaths attributable to IFIs ( $1 \%$ versus $4 \% ; \mathrm{P}=0.046$ ). 


\section{TABLE 2}

Posaconazole ( $800 \mathrm{mg} / \mathrm{day}$ ) therapy for uncommon invasive filamentous fungal infections

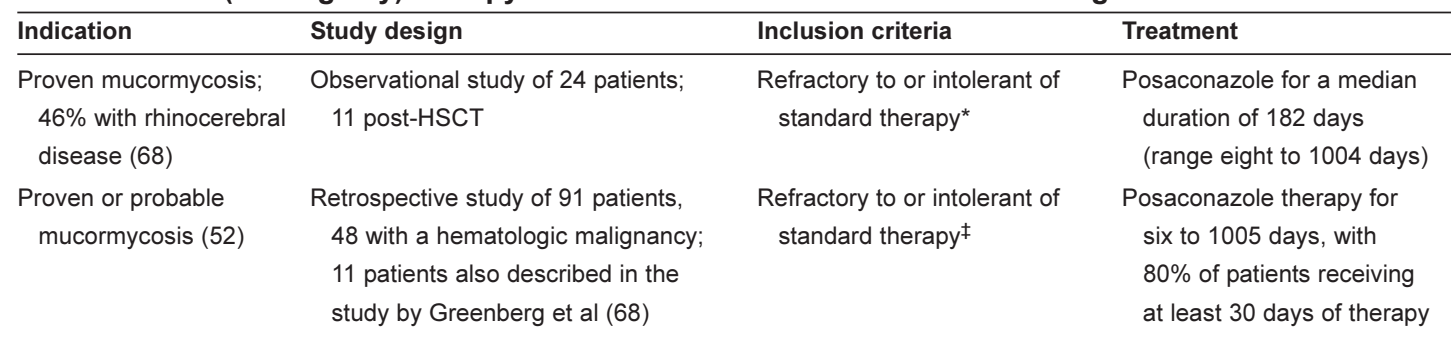

\section{Proven or probable} invasive fusariosis (24)
Retrospective analysis of 21 patients enrolled in three open-label clinical trials; $76 \%$ with a hematologic malignancy
Culture-proven phaeohyphomycosis $(69,70)$
Case reports: patient 1 - disseminated Exophiala spinifera infection; patient 2 - Alternaria species maxillary osteomyelitis
Proven eumycetoma or chromoblastomycosis (71)

Disseminated/pulmonary histoplasmosis (72)
Open-label, observational study of
12 patients

Observational study of six patients, three with HIV disease, two receiving chronic corticosteroid therapy and one with no identifiable immunosuppression

$\begin{array}{ll}\begin{array}{l}\text { Chronic pulmonary or } \\ \text { nonmeningeal }\end{array} & \begin{array}{c}\text { Open-label, phase II study of } \\ \text { disseminated } \\ \text { coccidioidomycosis } \\ \text { (53) }\end{array} \\ \begin{array}{l}\text { and three receiving corticosteroid } \\ \text { therapy }\end{array} \\ \begin{array}{l}\text { disseminated } \\ \text { coccidioidomycosis (73) }\end{array} & \begin{array}{l}\text { one each post-HSCT and receiving } \\ \text { two with diabetes mellitus and }\end{array} \\ & \text { corticosteroid therapy }\end{array}$

Refractory to or intolerant of standard therapy§

\section{Posaconazole for up to} 12 months
Patient 1 - refractory to months Patient 1 - posaconazole for of therapy with amphotericin B and itraconazole over several years; patient 2 - refractory to voriconazole, caspofungin, amphotericin $\mathrm{B}$, ketoconazole, fluconazole and terbinafine over a 12-year period

Refractory to standard therapy ${ }^{\dagger \dagger}$ Posaconazole for 168 to 1015 days

\section{Refractory to or intolerant of Posaconazole for six to standard therapy $¥ \ddagger \quad 34$ weeks}

\section{Results \\ response in $79 \%$ \\ Complete or partial response 12 weeks after initiation of therapy in $60 \%$, regardless of adjunctive surgical debridement $^{\dagger}$ \\ Complete or partial response in $48 \%$, although less so in the subset of patients with disseminated infection, persistent neutropenia, or prior HSCTI}

Clinical and mycological suppression in both cases; ongoing posaconazole administration required to prevent the re-emergence of active lesions in both patients**

Complete or partial response in 10 patients at treatment end

Complete or partial response in all six patients by treatment end; no no long-term follow-up provided

$\begin{array}{lc}\begin{array}{l}\text { Primary therapy in } 70 \% \text { of } \\ \text { patients; } 30 \% \text { had relapsing }\end{array} & \begin{array}{c}\text { Posaconazole (400 mg/day) } \\ \text { for a median duration }\end{array} \\ \begin{array}{l}\text { disease but were not receiving } \\ \text { other antifungal therapy at }\end{array} & \begin{array}{l}173 \text { days (range } 15 \text { to } \\ \text { study entry }\end{array} \\ \begin{array}{l}\text { Refractory to amphotericin B } \\ \text { and/or an azole for a minimum }\end{array} & \begin{array}{l}\text { Posaconazole for } 34 \text { to } \\ \text { duration of } 40 \text { days and a } 12 \text { patients }\end{array} \\ \text { median duration of } 306 \text { days } & \text { received the drug for } \\ \end{array}$

Satisfactory response in $85 \%$ at week 24 ; relapse in three of nine patients after treatment end§§

Complete or partial response in $73 \%$

${ }^{*}$ For the majority of patients, standard therapy included amphotericin B, with or without itraconazole or caspofungin. Two patients received monotherapy with other agents (itraconazole and voriconazole); †The partial and complete response rate following amphotericin B therapy was documented at $60 \%$ (74); $¥$ Eighty-five per cent received lipid formulations of amphotericin B, $26 \%$ amphotericin B deoxycholate and $31 \%$ voriconazole. Patients may have received more than one antifungal agent; $\S$ Twenty patients were initially treated with amphotericin B, with or without itraconazole and/or caspofungin, for a median of eight days (range two to 38 days). One had not received any prior antifungal therapy; "A complete or partial response was noted in five of 11 patients (45\%) with fusariosis treated with voriconazole, although details regarding dose and duration of treatment, as well as neutrophil recovery, are lacking (75); **Five cases of phaeohyphomycosis due to Alternaria, Bipolaris and Exophiala species were reported to be successfully treated with voriconazole, although duration of therapy and adjunctive surgical procedures were not documented (75); ${ }^{\dagger}$ Standard therapy included itraconazole, ketoconazole, terbinafine or flucytosine for a cumulative duration of two months to eight years; $¥ \neq P$ atients had received antifungal therapy with either fluconazole, itraconazole, voriconazole or amphotericin B for a variable duration (range eight days to 3.5 years) before study entry; $\S \S$ Satisfactory response was defined as a $50 \%$ reduction from baseline in the Mycoses Study Group score, a composite evaluation of symptoms, physical examination findings and laboratory investigations. HSCT Hematopoietic stem cell transplant. Data from references 24, $52,53,68$ to 73

In both studies, breakthrough infections during posaconazole therapy were most commonly caused by Candida and Aspergillus species. The effect of posaconazole prophylaxis on the susceptibility patterns of these fungi is not yet known. Furthermore, there have been no studies comparing posaconazole with voriconazole, which has a similar spectrum of activity, in terms of primary prophylaxis in this patient population. Nonetheless, recent guidelines from the Infectious Diseases Society of America (54) recommend posaconazole for the prevention of invasive aspergillosis in both populations; posaconazole has been approved for use in Canada for the prophylaxis of Aspergillus and Candida species infections in patients 13 years of 
age and older who are at high-risk for these infections, either from prolonged neutropenia or following HSCT (10).

\section{RESISTANCE MECHANISMS}

Resistance to the triazoles arises either from mutations in the gene (ERG11) encoding the target enzyme (CYP51) or from the overexpression of efflux pumps. The long side chain of posaconazole is thought to reduce the development of resistance by either mechanism. Mutations in the active site of CYP51, sufficient to render C albicans resistant to fluconazole and voriconazole, do not alone create posaconazole resistance. Instead, numerous mutations outside of the active site are required to disrupt multiple secondary interactions between the posaconazole side chain and CYP51 $(55,56)$. Furthermore, posaconazole is not a substrate for the MDR1 or FLU1 efflux pumps, and is a poor substrate for the CDR1 and CDR2 efflux pumps. In one study, $\mathrm{C}$ albicans isolates remained susceptible to posaconazole despite a 30 -fold increase in the expression of the CDR2 pump. As a result, of 24 fluconazole-resistant clinical isolates of $\mathrm{C}$ albicans, 17 had MICs greater than $1 \mathrm{mg} / \mathrm{L}$ for voriconazole, seven for itraconazole and only one for posaconazole. Overexpression of ERG11, which has been shown to confer resistance to fluconazole, is another possible contributor to posaconazole resistance, although this has yet to be demonstrated in vitro (57).

Clinically, posaconazole has been used successfully to treat infections resulting from fluconazole- or itraconazoleresistant Candida species (42). Its high barrier to resistance is also apparent in studies (58) of azole-resistant Aspergillus species, in which itraconazole- or voriconazole-resistant isolates exhibited only low-level cross-resistance to posaconazole, and amphotericin-resistant isolates exhibited no cross-resistance.

As a consequence of increasing resistance to antifungal agents and the attendant possibility of resulting clinical failures, interest in antifungal drug combinations has grown. No antagonism was observed between posaconazole and caspofungin against $C$ glabrata in in vitro studies, nor between posaconazole and amphotericin $\mathrm{B}$ against $\mathrm{C}$ albicans and $\mathrm{C}$ neoformans in in vivo or in vitro studies, respectively (59-61). With the latter combination, either additive or synergistic effects were observed for the majority of $\mathrm{C}$ neoformans isolates tested. Moreover, no antagonism was observed between posaconazole and caspofungin in a murine model of invasive aspergillosis (62). Although there have been no prospective clinical trials reported to date of posaconazole use in combination with other antifungal agents, these studies suggest that no deleterious effects would be expected by coadminstration of agents from two different classes. Notably, however, in the presence of a fully susceptible organism, no additional therapeutic benefit would necessarily be derived from the combination.

\section{SAFETY AND TOLERABILITY}

The commonly reported adverse effects of posaconazole are similar to those of other triazoles. Adverse events were reported in 38\% of 428 patients, 109 of whom received posaconazole for longer than six months, and 27 of whom received it for longer than 12 months (29). Adverse events lead to discontinuation of therapy in only $6 \%$ of patients, and there was no difference in the type or incidence of adverse events reported among patients receiving therapy for longer or shorter than six months. The most commonly reported adverse events were nausea, which occurred in $8 \%$ of patients, and vomiting, which occurred in $6 \%$ of patients. Abdominal pain, diarrhea, headache, fatigue, dizziness and rash were also each reported in $2 \%$ to $4 \%$ of patients. Serious adverse events occurred in $8 \%$ of patients, and included elevations in serum hepatic aminotransferases and serum creatinine in $2 \%$ of patients, and prolongation of the corrected QT interval in $1 \%$. QT prolongation occurred between 28 and 64 days of therapy. A similar rate and spectrum of adverse events has been reported with the three times daily dosing regimen (18). In total, the safety of posaconazole has been evaluated in 1844 patients and was found to be similar to fluconazole in comparative studies (10). Monitoring of serum aminotransferases and markers of liver function is recommended before the initiation of therapy, and intermittently throughout, because there have been rare reports of fatal cholestasis and hepatic failure associated with posaconazole use (10).

\section{CONCLUSIONS}

Despite the diverse spectrum of activity indicated by in vitro and in vivo experimental data, posaconazole has received Canadian approval only for the treatment of OPC and refractory invasive aspergillosis, as well as for the prophylaxis of invasive aspergillosis and candidiasis in high-risk patients (10). Given that fluconazole is an inexpensive, well-tolerated, long-used option for most patients with HIV-associated OPC, posaconazole will most likely be used only in those patients with azole-refractory disease. In this setting, its oral formulation provides benefit over intravenous caspofungin, the other approved treatment option for these patients. Likewise, in patients with refractory invasive aspergillosis, oral posaconazole may be of benefit over intravenous caspofungin. Posaconazole may be of use even in those patients who have failed therapy with voriconazole or who are infected with voriconazoleresistant isolates, because the risk of cross-resistance appears to be low. Nonetheless, close monitoring of susceptibility patterns is indicated. In high-risk patients with hematological malignancies, including those undergoing induction chemotherapy for acute leukemia, hematopoietic stem cell transplantation and treatment for severe graft-versus-host disease, posaconazole demonstrated a reduction in all-cause mortality compared with fluconazole, primarily on the basis of its activity against Aspergillus species. Posaconazole is now the recommended prophylactic regimen for use in these high-risk patients, but its cost may limit its use. As recommended in the Infectious Diseases Society of America guidelines, further studies may be helpful to better define the precise population at highest risk. In addition, increasing use requires close laboratory monitoring for the development of resistance, as well as for an increased incidence of those IFIs against which posaconazole is less effective, including fusariosis and scedosporiosis. In terms of other IFIs, with its well-tolerated oral formulation and few drug interactions, posaconazole may become the agent of choice for long-term treatment of the opportunistic mycoses against which voriconazole has little or no activity, including mucormycosis. Again, the impact of this projected increase in posaconazole use on fungal susceptibility patterns needs to be monitored closely. However, the high barrier to resistance created by posaconazole's unique structure may secure its niche in treating polyene-, echinocandin- and even triazole-resistant IFIs. 


\section{REFERENCES}

1. Marr KA, Seidel K, White TC, Bowden RA. Candidemia in allogeneic blood and marrow transplant recipients: Evolution of risk factors after the adoption of prophylactic fluconazole. J Infect Dis 2000;181:309-16.

2. Pagano L, Caira M, Nosari A, et al. Fungal infections in recipients of hematopoietic stem cell transplants: Results of the SEIFEM B-2004 study - Sorveglianza Epidemiologica Infezioni Fungine Nelle Emopatie Maligne. Clin Infect Dis 2007;45:1161-70.

3. Ullmann AJ, Sanz MA, Tramarin A, et al; Longitudinal Evaluation of Antifungal Drugs (LEAD I) Investigators. Prospective study of amphotericin B formulations in immunocompromised patients in 4 European countries. Clin Infect Dis 2006;43:e29-38.

4. Hachem RY, Kontoyiannis DP, Boktour MR, et al. Aspergillus terreus: An emerging amphotericin B-resistant opportunistic mold in patients with hematologic malignancies. Cancer 2004;101:1594-600.

5. Trifilio SM, Bennett CL, Yarnold PR, et al. Breakthrough zygomycosis after voriconazole administration among patients with hematologic malignancies who receive hematopoietic stem-cell transplants or intensive chemotherapy. Bone Marrow Transplant 2007;39:425-9.

6. Boucher HW, Groll AH, Chiou CC, Walsh TJ. Newer systemic antifungal agents: Pharmacokinetics, safety and efficacy. Drugs 2004;64:1997-2020.

7. Kahn JN, Garcia-Effron G, Hsu MJ, Park S, Marr KA, Perlin DS. Acquired echinocandin resistance in a Candida krusei isolate due to modification of glucan synthase. Antimicrob Agents Chemother 2007;51:1876-8.

8. Laverdière M, Lalonde RG, Baril JG, Sheppard DC, Park S, Perlin DS. Progressive loss of echinocandin activity following prolonged use for treatment of Candida albicans oesophagitis. J Antimicrob Chemother 2006;57:705-8.

9. Baixench MT, Aoun N, Desnos-Ollivier M, et al. Acquired resistance to echinocandins in Candida albicans: Case report and review. J Antimicrob Chemother 2007;59:1076-83.

10. Schering-Plough Canada Inc. Spriafil (posaconazole) product monograph, 2007.

11. European Medicines Agency (EMEA). EPARs for authorised medicinal products for human use. <http://www.emea.europa.eu/ htms/human/epar/eparintro.htm $>$ (Version current at June 5, 2008).

12. Munayyer HK, Mann PA, Chau AS, et al. Posaconazole is a potent inhibitor of sterol 14alpha-demethylation in yeasts and molds. Antimicrob Agents Chemother 2004;48:3690-6.

13. Courtney R, Pai S, Laughlin M, Lim J, Batra V. Pharmacokinetics, safety, and tolerability of oral posaconazole administered in single and multiple doses in healthy adults. Antimicrob Agents Chemother 2003;47:2788-95.

14. Courtney R, Wexler D, Radwanski E, Lim J, Laughlin M. Effect of food on the relative bioavailability of two oral formulations of posaconazole in healthy adults. Br J Clin Pharmacol 2003; $57: 218-22$.

15. Ezzet F, Wexler D, Courtney R, Krishna G, Lim J, Laughlin M. Oral bioavailability of posaconazole in fasted healthy subjects: Comparison between three regimens and basis for clinical dosage recommendations. Clin Pharmacokinet 2005;44:211-20.

16. Pfizer Canada Inc. Vfend (voriconazole) product monograph, 2006.

17. Cornely OA, Maertens J, Winston DJ, et al. Posaconazole vs. fluconazole or itraconazole prophylaxis in patients with neutropenia. N Engl J Med 2007;356:348-59.

18. Ullmann AJ, Lipton JH, Vesole DH, et al. Posaconazole or fluconazole for prophylaxis in severe graft-versus-host disease. N Engl J Med 2007;356:335-47. (Erratum in 2007;357:428).

19. Walsh TJ, Raad I, Patterson TF, et al. Treatment of invasive aspergillosis with posaconazole in patients who are refractory to or intolerant of conventional therapy: An externally controlled trial. Clin Infect Dis 2007;44:2-12.

20. Krieter P, Flannery B, Musick T, Gohdes M, Martinho M, Courtney R. Disposition of posaconazole following single-dose oral administration in healthy subjects. Antimicrob Agents Chemother 2004;48:3543-51.

21. Perfect JR, Cox GM, Dodge RK, Schell WA. In vitro and in vivo efficacies of the azole SCH56592 against Cryptococcus neoformans. Antimicrob Agents Chemother 1996;40:1910-3.

22. Imai JK, Singh G, Clemons KV, Stevens DA. Efficacy of posaconazole in a murine model of central nervous system aspergillosis. Antimicrob Agents Chemother 2004;48:4063-6. (Erratum in 2004;48:4931).

23. Pitisuttithum P, Negroni R, Graybill JR, et al. Activity of posaconazole in the treatment of central nervous system fungal infections. J Antimicrob Chemother 2005;56:745-55.

24. Raad II, Hachem RY, Herbrecht R, et al. Posaconazole as salvage treatment for invasive fusariosis in patients with underlying hematologic malignancy and other conditions. Clin Infect Dis 2006;42:1398-403.

25. Courtney R, Sansone A, Smith W, et al. Posaconazole pharmacokinetics, safety, and tolerability in subjects with varying degrees of chronic renal disease. J Clin Pharmacol 2005;45:185-92.

26. Sansone-Parsons A, Krishna G, Simon J, et al. Effects of age, gender, and race/ethnicity on the pharmacokinetics of posaconazole in healthy volunteers. Antimicrob Agents Chemother 2007;51:495-502.

27. Krishna G, Sansone-Parsons A, Martinho M, Kantesaria B, Pedicone L. Posaconazole plasma concentrations in juvenile patients with invasive fungal infection. Antimicrob Agents Chemother 2007;51:812-8.

28. Saad AH, DePestel DD, Carver PL. Factors influencing the magnitude and clinical significance of drug interactions between azole antifungals and select immunosuppressants. Pharmacotherapy 2006;26:1730-44.

29. Raad II, Graybill JR, Bustamante AB, et al. Safety of long-term oral posaconazole use in the treatment of refractory invasive fungal infections. Clin Infect Dis 2006;42:1726-34.

30. Wexler D, Courtney R, Richards W, Banfield C, Lim J, Laughlin M. Effect of posaconazole on cytochrome P450 enzymes: A randomized, open-label, two-way crossover study. Eur J Pharm Sci 2004;21:645-53.

31. Sabatelli F, Patel R, Mann PA, et al. In vitro activities of posaconazole, fluconazole, itraconazole, voriconazole, and amphotericin B against a large collection of clinically important molds and yeasts. Antimicrob Agents Chemother 2006;50:2009-15.

32. Sóczó G, Kardos G, McNicholas PM, et al. Correlation of posaconazole minimum fungicidal concentration and time-kill test against nine Candida species. J Antimicrob Chemother 2007;60:1004-9.

33. Paphitou NI, Ostrosky-Zeichner L, Paetznick VL, Rodriguez JF, Chen E, Rex JH. In vitro antifungal susceptibilities of Trichosporon species. Antimicrob Agents Chemother 2002;46:1144-6.

34. Lutz JE, Clemons KV, Aristizabal BH, Stevens DA. Activity of the triazole SCH 56592 against disseminated murine coccidioidomycosis. Antimicrob Agents Chemother 1997;41:1558-61.

35. Espinel-Ingroff A. Comparison of in vitro activities of the new triazole SCH56592 and the echinocandins MK-0991 (L-743,872) and LY303366 against opportunistic filamentous and dimorphic fungi and yeasts. J Clin Microbiol 1998;36:2950-6.

36. Sugar AM, Liu XP. In vitro and in vivo activities of SCH 56592 against Blastomyces dermatitidis. Antimicrob Agents Chemother 1996;40:1314-6.

37. Barchiesi F, Arzeni D, Camiletti V, et al. In vitro activity of posaconazole against clinical isolates of dermatophytes. J Clin Microbiol 2001;39:4208-9.

38. Pfaller MA, Messer SA, Boyken L, et al. In vitro activities of voriconazole, posaconazole, and fluconazole against 4,169 clinical isolates of Candida spp. and Cryptococcus neoformans collected during 2001 and 2002 in the ARTEMIS global antifungal surveillance program. Diagn Microbiol Infect Dis 2004;48:201-5.

39. Diekema DJ, Messer SA, Hollis RJ, Jones RN, Pfaller MA. Activities of caspofungin, itraconazole, posaconazole, ravuconazole, voriconazole, and amphotericin B against 448 recent clinical isolates of filamentous fungi. J Clin Microbiol 2003;41:3623-6.

40. Groll AH, Walsh TJ. Antifungal efficacy and pharmacodynamics of posaconazole in experimental models of invasive fungal infections. Mycoses 2006;49(Suppl 1):7-16.

41. Vazquez JA, Skiest DJ, Nieto L, et al. A multicenter randomized trial evaluating posaconazole versus fluconazole for the treatment of oropharyngeal candidiasis in subjects with HIV/AIDS. Clin Infect Dis 2006;42:1179-86.

42. Skiest DJ, Vazquez JA, Anstead GM, et al. Posaconazole for the treatment of azole-refractory oropharyngeal and esophageal candidiasis in subjects with HIV infection. Clin Infect Dis 2007;44:607-14. 
43. Goldman M, Cloud GA, Wade KD, et al. A randomized study of the use of fluconazole in continuous versus episodic therapy in patients with advanced HIV infection and a history of oropharyngeal candidiasis: AIDS Clinical Trials Group study 323/Mycoses Study Group study 40. Clin Infect Dis 2005;41:1473-80.

44. Barchiesi F, Maracci M, Radi B, et al. Point prevalence, microbiology and fluconazole susceptibility patterns of yeast isolates colonizing the oral cavities of HIV-infected patients in the era of highly active antiretroviral therapy. J Antimicrob Chemother 2002;50:999-1002.

45. Kartsonis NA, Saah A, Lipka CJ, Taylor A, Sable CA. Second-line therapy with caspofungin for mucosal or invasive candidiasis: Results from the caspofungin compassionate-use study. J Antimicrob Chemother 2004;53:878-81.

46. Vazquez JA, Skiest DJ, Tissot-Dupont H, Lennox JL, Boparai N, Isaacs R. Safety and efficacy of posaconazole in the long-term treatment of azole-refractory oropharyngeal and esophageal candidiasis in patients with HIV infection. HIV Clin Trials 2007;8:86-97.

47. Vehreschild JJ, Krüger K, Kurzai O, et al. Salvage therapy of refractory chronic disseminated candidiasis in a patient with acute myeloid leukaemia and secondary prophylaxis during allogeneic stem cell transplantation. Mycoses 2006;49(Suppl 1):42-7.

48. Schilling A, Seibold M, Mansmann V, Gleissner B. Successfully treated Candida krusei infection of the lumbar spine with combined caspofungin/posaconazole therapy. Med Mycol 2008;46:79-83.

49. Raad II, Hanna HA, Boktour M, et al. Novel antifungal agents as salvage therapy for invasive aspergillosis in patients with hematologic malignancies: Posaconazole compared with high-dose lipid formulations of amphotericin B alone or in combination with caspofungin. Leukemia 2008;22:496-503.

50. Maertens J, Glasmacher A, Herbrecht R, et al; Caspofungin Combination Therapy Study Group. Multicenter, noncomparative study of caspofungin in combination with other antifungals as salvage therapy in adults with invasive aspergillosis. Cancer 2006;107:2888-97.

51. Mullane K, Toor AA, Kalnicky C, Rodriguez T, Klein J, Stiff P. Posaconazole salvage therapy allows successful allogeneic hematopoietic stem cell transplantation in patients with refractory invasive mold infections. Transpl Infect Dis 2007;9:89-96.

52. van Burik JA, Hare RS, Solomon HF, Corrado ML, Kontoyiannis DP. Posaconazole is effective as salvage therapy in zygomycosis: A retrospective summary of 91 cases. Clin Infect Dis 2006;42:e61-5. (Erratum in 2006;43:1376).

53. Catanzaro A, Cloud GA, Stevens DA, et al. Safety, tolerance, and efficacy of posaconazole therapy in patients with nonmeningeal disseminated or chronic pulmonary coccidioidomycosis. Clin Infect Dis 2007:45:562-8.

54. Walsh TJ, Anaissie EJ, Denning DW, et al; Infectious Diseases Society of America. Treatment of aspergillosis: Clinical practice guidelines of the Infectious Diseases Society of America. Clin Infect Dis 2008;46:327-60.

55. Xiao L, Madison V, Chau AS, Loebenberg D, Palermo RE, McNicholas PM. Three-dimensional models of wild-type and mutated forms of cytochrome P450 14alpha-sterol demethylases from Aspergillus fumigatus and Candida albicans provide insights into posaconazole binding. Antimicrob Agents Chemother 2004; 48:568-74.

56. Li X, Brown N, Chau AS, et al. Changes in susceptibility to posaconazole in clinical isolates of Candida albicans. J Antimicrob Chemother 2004;53:74-80.
57. Chau AS, Mendrick CA, Sabatelli FJ, Loebenberg D, McNicholas PM. Application of real-time quantitative PCR to molecular analysis of Candida albicans strains exhibiting reduced susceptibility to azoles. Antimicrob Agents Chemother 2004:48:2124-31.

58. Manavathu EK, Cutright JL, Loebenberg D, Chandrasekar PH. A comparative study of the in vitro susceptibilities of clinical and laboratory-selected resistant isolates of Aspergillus spp. to amphotericin B, itraconazole, voriconazole, and posaconazole (SCH 56592). J Antimicrob Chemother 2000;46:229-34.

59. Oliveira ER, Fothergill AW, Kirkpatrick WR, Coco BJ, Patterson TF, Redding SW. In vitro interaction of posaconazole and caspofungin against clinical isolates of Candida glabrata. Antimicrob Agents Chemother 2005;49:3544-5.

60. Barchiesi F, Schimizzi AM, Caselli F, et al. Interactions between triazoles and amphotericin B against Cryptococcus neoformans. Antimicrob Agents Chemother 2000;44:2435-41.

61. Cacciapuoti A, Gurnani M, Halpern J, Norris C, Patel R, Loebenberg D. Interaction between posaconazole and amphotericin B in concomitant treatment against Candida albicans in vivo. Antimicrob Agents Chemother 2005;49:638-42.

62. Cacciapuoti A, Halpern J, Mendrick C, Norris C, Patel R, Loebenberg D. Interaction between posaconazole and caspofungin in concomitant treatment of mice with systemic Aspergillus infection. Antimicrob Agents Chemother 2006;50:2587-90.

63. Keating GM. Posaconazole. Drugs 2005;65:1553-67.

64. Metcalf SC, Dockrell DH. Improved outcomes associated with advances in therapy for invasive fungal infections in immunocompromised hosts. J Infect 2007;55:287-99.

65. Pfaller MA, Diekema DJ. Rare and emerging opportunistic fungal pathogens: Concern for resistance beyond Candida albicans and Aspergillus fumigatus. J Clin Microbiol 2004:42:4419-31.

66. Cappelletty D, Eiselstein-McKitrick K. The echinocandins. Pharmacotherapy 2007;27:369-88.

67. Revankar SG. Dematiaceous fungi. Mycosis 2007;50:91-101

68. Greenberg RN, Mullane K, van Burik JH, et al. Posaconazole as salvage therapy for zygomycosis. Antimicrob Agents Chemother 2006;50:126-33.

69. Negroni R, Helou SH, Petri N, Robles AM, Arechavala A, Bianchi MH. Case study: Posaconazole treatment of disseminated phaeohyphomycosis due to Exophiala spinifera. Clin Infect Dis 2004;38:e15-20.

70. Proia LA, Trenholme GM. Chronic refractory phaeohyphomycosis: Successful treatment with posaconazole. Mycoses 2006;49:519-22.

71. Negroni R, Tobón A, Bustamante B, Shikanai-Yasuda MA, Patino $\mathrm{H}$, Restrepo A. Posaconazole treatment of refractory eumycetoma and chromoblastomycosis. Rev Inst Med Trop Sao Paulo 2005;47:339-46.

72. Restrepo A, Tobón A, Clark B, et al. Salvage treatment of histoplasmosis with posaconazole. J Infect 2007;54:319-27.

73. Stevens DA, Rendon A, Gaona-Flores V et al. Posaconazole therapy for refractory coccidioidomycosis. Chest 2007;132:952-8.

74. Herbrecht R, Letscher-Bru V, Bowden RA, et al. Treatment of 21 cases of invasive mucormycosis with amphotericin B colloidal dispersion. Eur J Clin Microbiol Infect Dis 2001;20:460-6.

75. Perfect JR, Marr KA, Walsh TJ, et al. Voriconazole treatment for less-common, emerging, or refractory fungal infections. Clin Infect Dis 2003;36:1122-31. 


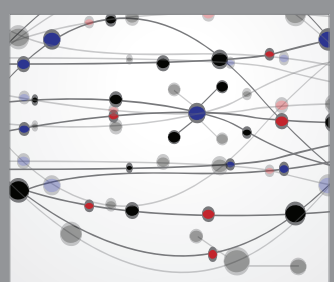

The Scientific World Journal
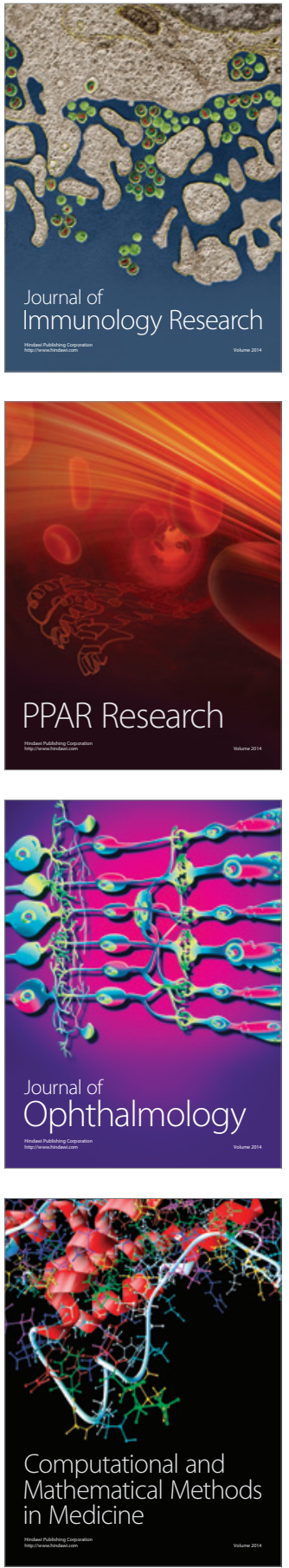

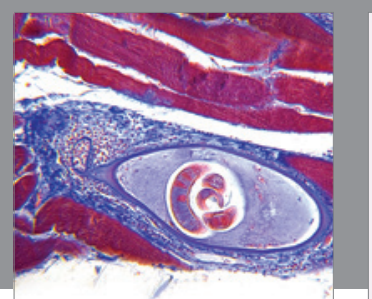

Gastroenterology Research and Practice

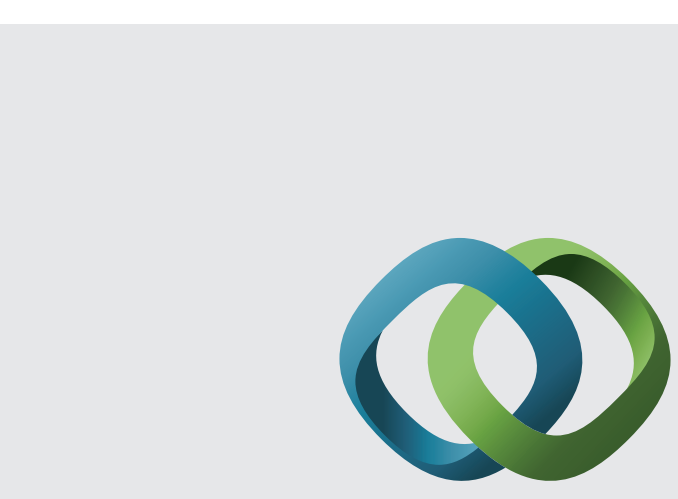

\section{Hindawi}

Submit your manuscripts at

http://www.hindawi.com
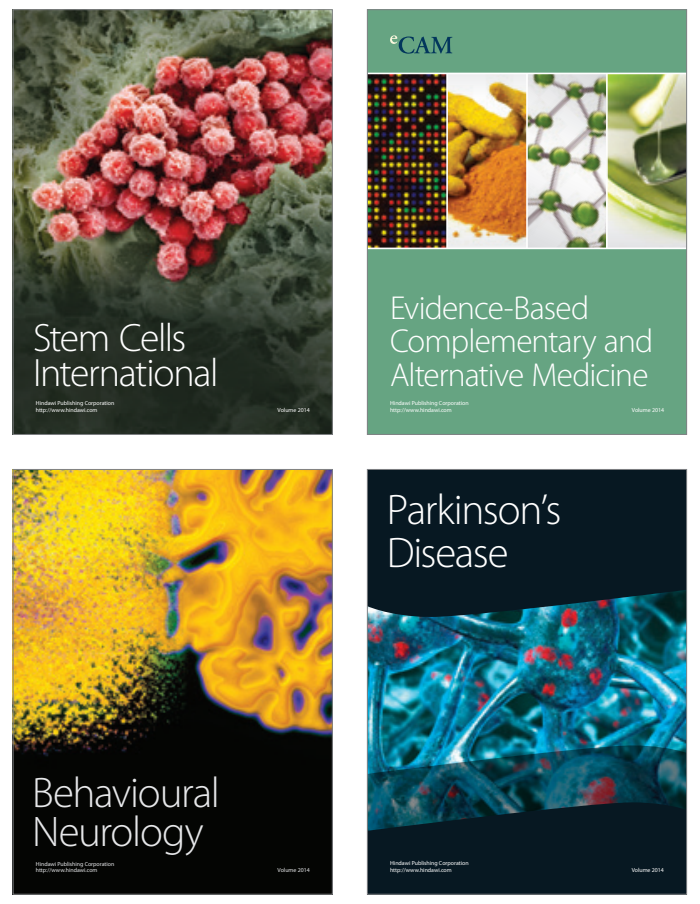
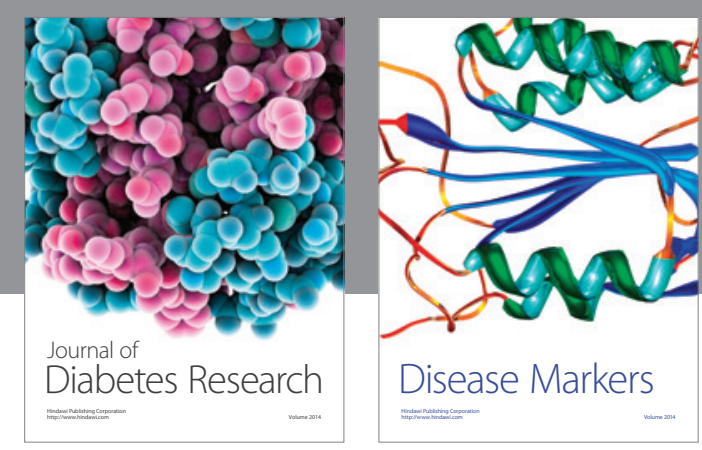

Disease Markers
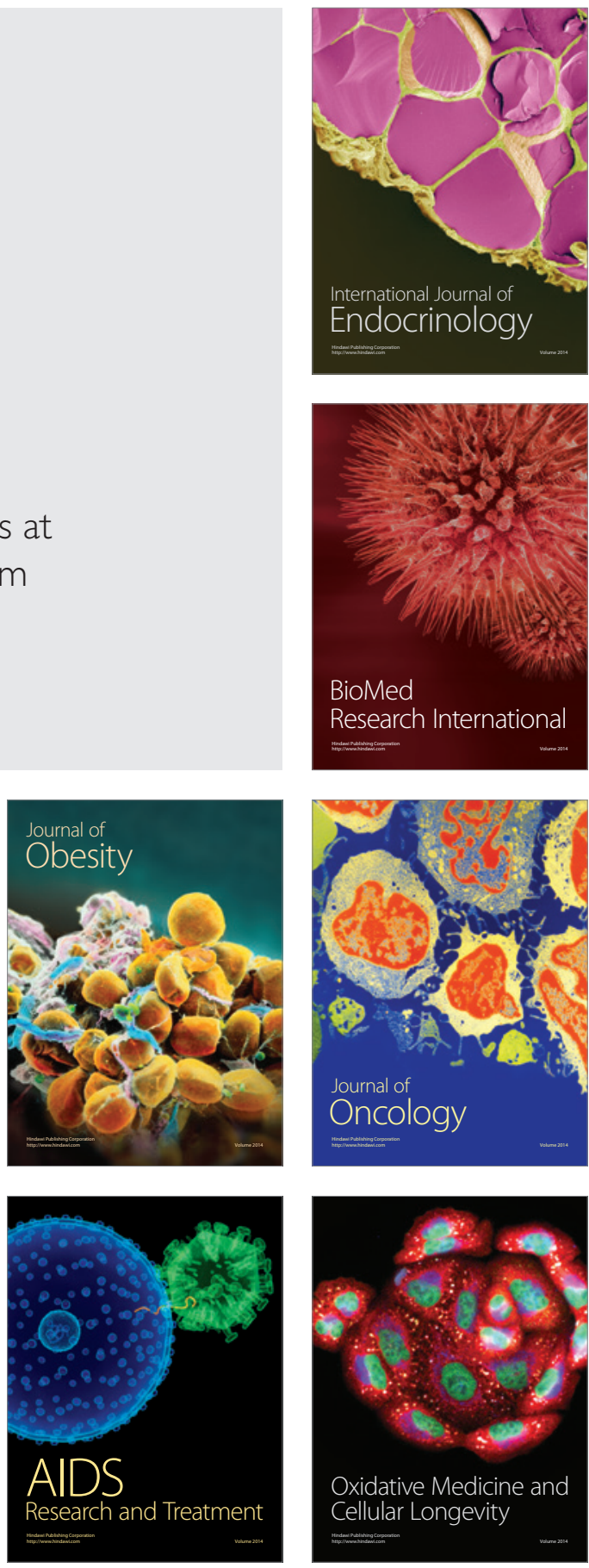